Tohokn J. exp. Med., 1980, 131, 7-13

\title{
Cerebro-Cortical Innervation of the Urinary Bladder
}

\author{
William E. Bradley \\ Department of Neurology, University of Minnesota Medical \\ School, Minneapolis, Minnesota 55455, U.S.A.
}

Bradley, W.E. Cerebro-Cortical Innervation of the Urinary Bladder. Tohoku J. exp. Med., 1980, 131 (1), 7-13 - In this report cerebro-cortical innervation of the urinary bladder has been investigated in man by the use of electroencephalography and in the cat by the application of the evoked potential technique. Electroencephalographic monitoring during bladder filling while the patient was asleep was a useful method to determine cerebral response to stimulation of bladder sensory receptors. Bladder filling was performed during stage IV sleep. In the intact individual detrusor reflex contraction or attainment of bladder capacity was followed by prompt arousal and desynchronization of EEG rhythms. In patients with interruption of bladder sensory pathways in the periphery or in the spinal cord there was loss of impairment of the EEG response to bladder filling. In the cat, anesthetized with sodium pentobarbital, electrical stimulation of the nerve supply to the urinary detrusor muscle and to the pudendal nerve innervation to the periurethral striated muscle and anal sphincter, evoked a diphasic response in the cerebral cortex of the frontal lobe. All responses were grouped in the same area and were of short latency. Similar potentials have been evoked in man. These studies demonstrate that the peripheral, spinal and cerebral innervation of the urinary bladder in man are definable by electrophysiologic techniques. As a result, these electrophysiologic techniques are applicable to defining neuropathic changes in the urinary bladder innervation in man.

sleep cystometry; reflex evoked potentials; autonomic neuropathy

Cerebro-cortical innervation and control of the urinary bladder is a relatively unexposed area in the experimental animal and man (Andrew and Nathan 1974; Bradley 1977). Understanding the nature of this control and development of clinical methods for detection of this impairment are a necessary prelude to the effective management of urinary incontinence occurring in such diseases as cerebrovascular disease, Parkinson's disease and dementia associated with aging.

Electrophysiological methods for demonstrating cerebro-cortical innervation of the lower urinary tract include stimulation of specific brain areas in the experimental animal, electroencephalography (Bradley 1977) and cerebro-cortical potentials evoked by stimulation of the lower urinary tract. The latter two methods offer the detection of disease in specific areas of the brain and spinal cord controlling urinary bladder function. 


\section{Materials and Methods}

For the measurement of electroencephalographic responses to bladder filling and detrusor reflex contraction, the patients had placement of scalp recording electrodes according to the 10-20 systems. Patients were sleep deprived during the night prior to recording. On the morning of the study, an indwelling urethral catheter was inserted and the patient allowed to sleep. With the attainment of stage IV sleep characterized by a predominance of high voltage delta activity, a gas cystometrogram was performed.

For the measurement of cerebral evoled potentials, cats were employed. The animals were anesthetized with intravenous pentobarbital and a tracheostomy tube inserted. A midline laparotomy incision was made and the pelvic nerves were exposed, and Sherrington type bipolar electrodes were applied and connected to an electrical stimulator. The pudendal nerves were exposed through a dorsal approach, and bipolar stimulating electrodes were applied to the pudendal urethral and pudendal anal nerves and connected to an electrical stimulator.

A frontal craniotomy was then performed and the cruciate sulcus exposed bilaterally (Fig. 1). Silver ball electrodes were used to record the cerebro-cortical evoked responses from identified areas along the cruciate sulcus. Responses were averaged by use of a computer of average transients.

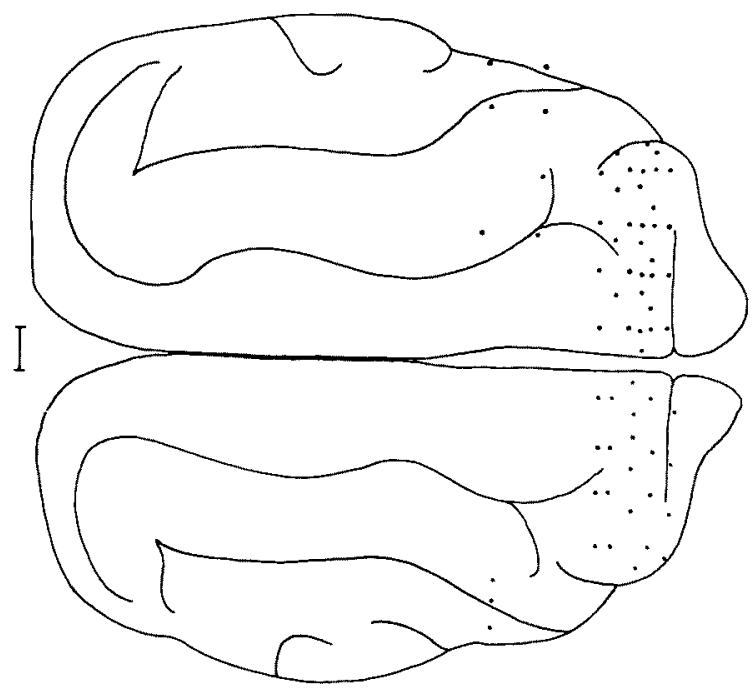

Fig. 1. Map of recording points indicated by dots located posterior to cruciate sulcus of cat cerebral cortex.

\section{Results}

\section{Evoked potentials}

Supramaximal stimulation of the pelvic detrusor nerve resulted in a bilateral diphasic positive negative response localized to a discrete portion of the sensory cortex in close promixity to the cruciate sulcus (Fig. 2). The diphasic response was observed on the medial surface of the hemisphere (Fig. 3). The latency or transit time for the response was slightly longer in the ipsilateral hemisphere than in the contralateral hemisphere. 


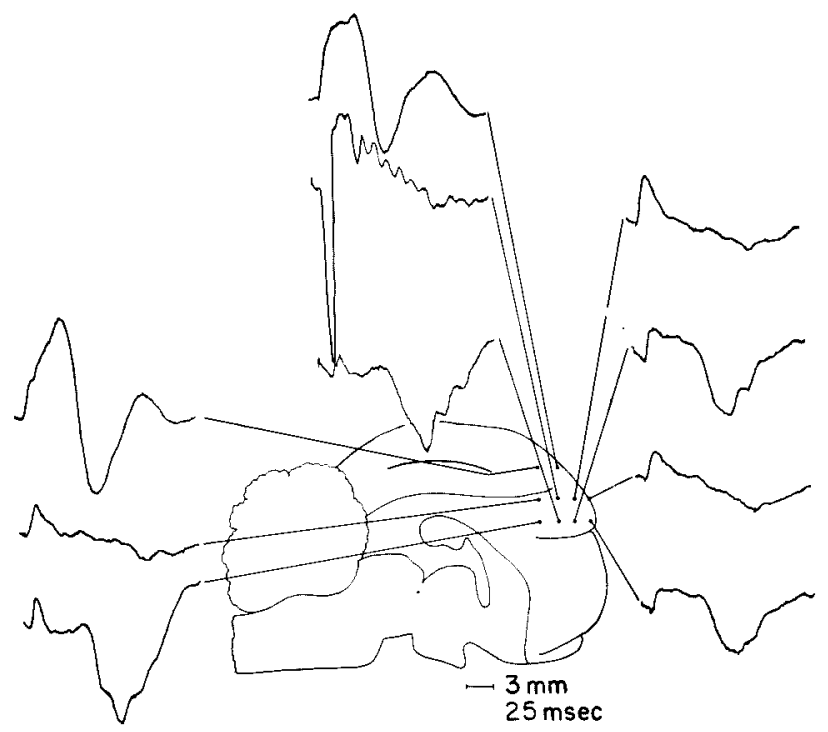

Fig. 2. Recordings of evoked responses from frontal lobes by stimulation of contralateral pelvic detrusor nerve.

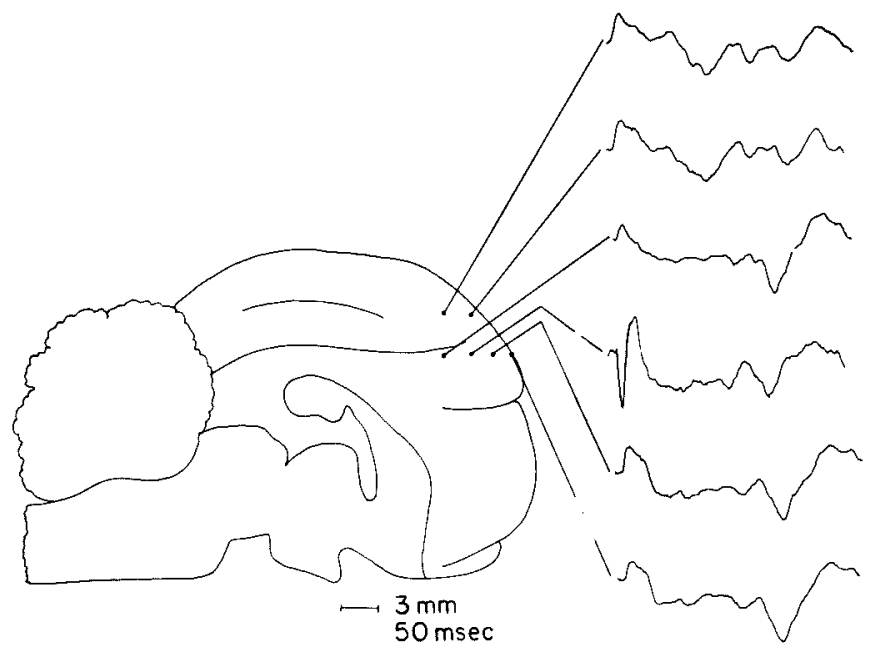

Fig. 3. Recordings of evoked responses from frontal lobes by stimulation of ipsilateral pelvic detrusor nerve.

Supramaximal stimulation of the pudendal urethral nerve evoked a discrete high amplitude diphasic response located in an area in close proximity to that observed for pelvic nerve stimulation (Fig. 4). This was present in both cerebral hemispheres and was increased in the ipsilateral hemisphere. With longer recording times a second diphasic response was observed (Fig. 5). 


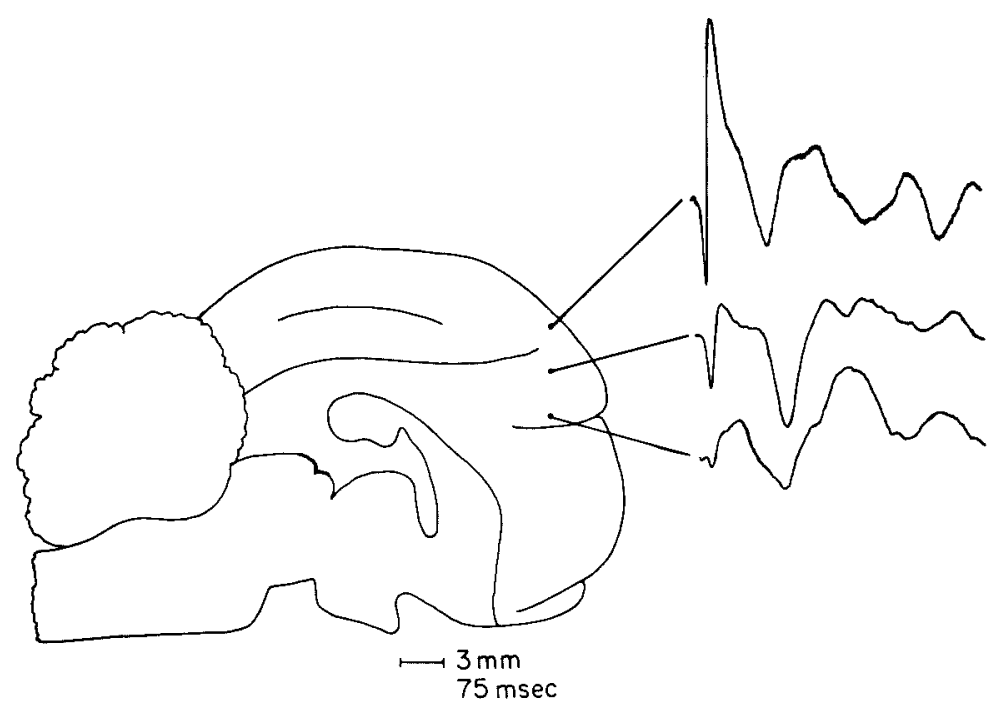

Fig. 4. Recordings of responses evoked from frontal lobe of cat by stimulation of contralateral pudendal urethral nerve.

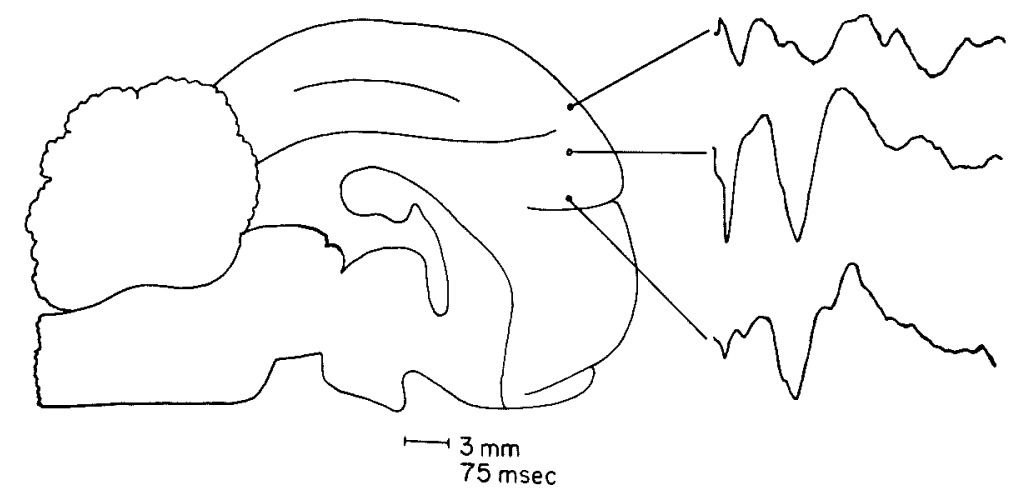

Fig. 5. Responses evoked from frontal lobe of eat by stimulation of ipsilateral pudendal urethral nerve.

Supramaximal stimulation of the pudendal anal nerve evoked a discrete diphasic initially surface positive wave in a similar location to that evoked by stimulation of the pudendal urethral nerve (Figs. 6 and 7).

Similar potentials have been evoked from the scalp of man in the central vertex region by stimulation of the lower urinary tract.

\section{Electroencephalography}

Sleep deprived cystometry was performed such that a cystometrogram was performed during deep sleep. In the normal individual as bladder capacity was 


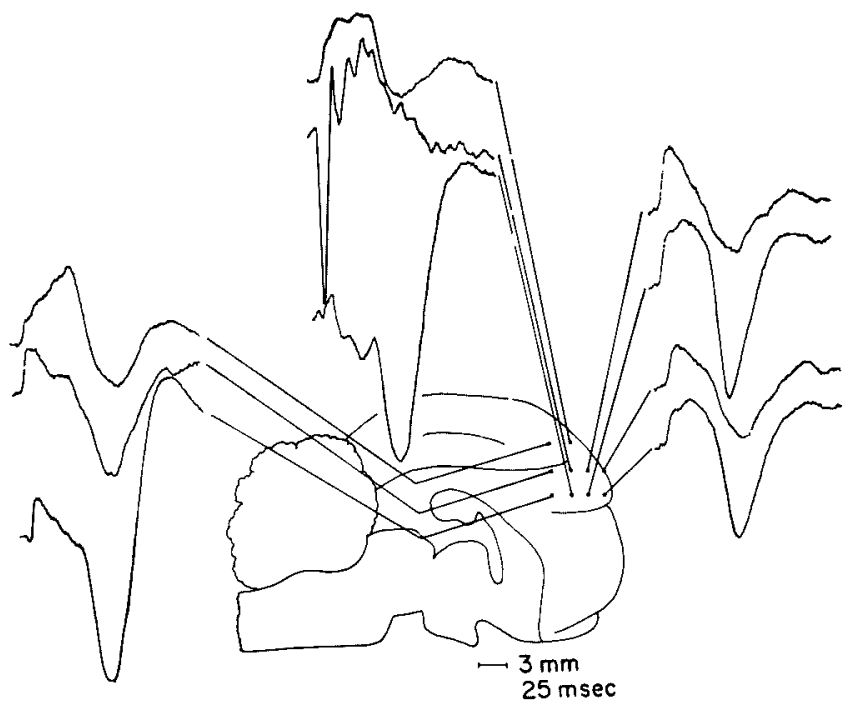

Fig. 6. Evoked responses from frontal lobes by stimulation of contralateral pudendal anal nerve.

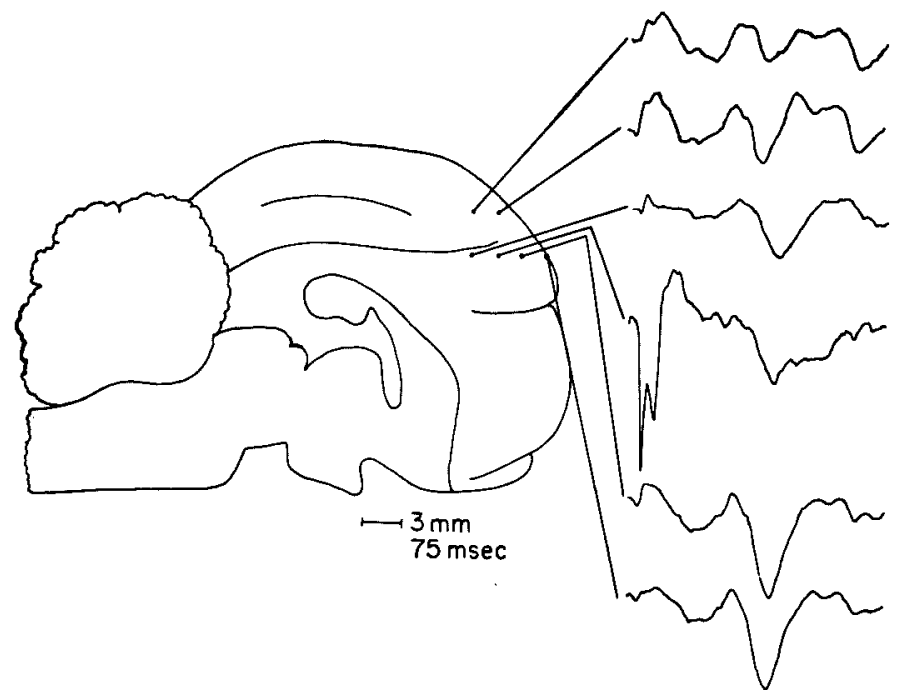

Fig. 7. Responses evoked in frontal lobe of cat by stimulation of ipsilateral pudendal anal nerve.

attained or if a detrusor reflex contraction occurred, there was rapid desynchronization of brain rhythms concurrent with arousal of the patient (Fig. 8).

However, in patients with interruption of the sensory nerves either in the periphery, in the spinal cord or in the brain there was no responses of the brain rhythms to bladder filling (Fig. 9). 

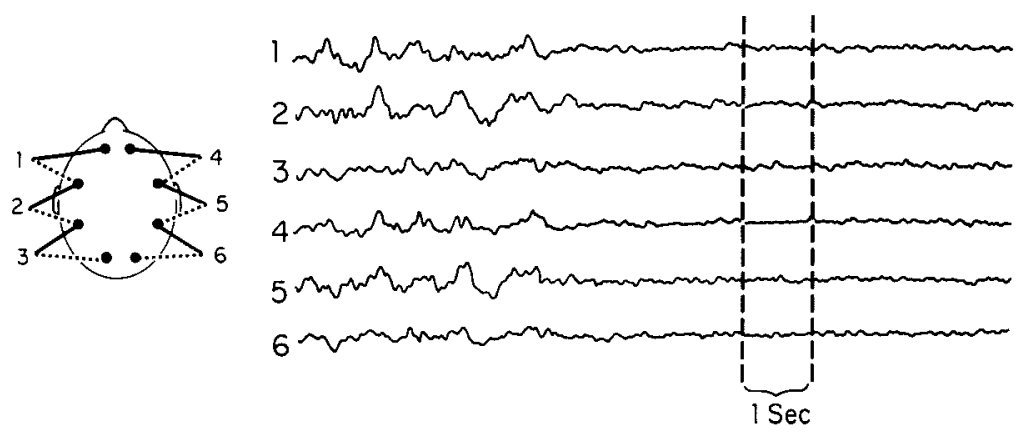

Fig. 8. Sleep EEG showing alteration of slow rhythms and arousal - black bar is representation of detrusor reflex contraction.

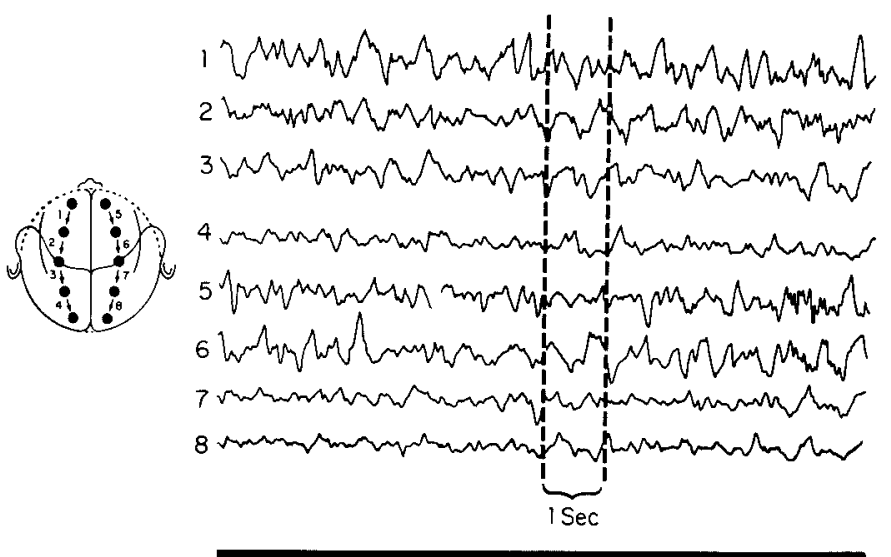

Fig. 9. Failure to alter brain rhythms during detrusor reflex contraction (black bar) in patient with interruption of cortical sensory pathways.

\section{Discussion}

Previous studies of electrical stimulation in the area of the cruciate sulcus of the cat (Gjone 1963) have demonstrated contractile responses to stimulation anterior to the cruciate sulcus. Destructive lesions in the experimental animal have also been observed to have an effect on the cystometrogram (Langworthy and Kolb 1933). Other effects demonstrating a brain-bladder interrelationship have also been observed (Imabayashi and Ohnuma 1977; Dill et al. 1977). However, this is the first reported study of direct anatomical connection from the bladder innervation of the cat to the cerebral cortex. The nature of the diphasic response is similar to that observed in the cerebellar cortex (Bradley and Teague 1969; Bradley and Scott 1978). This method when applied to humans offers a precise method for demonstrating the anatomic integrity of spinal cord and cortical pathways concerned with bladder and sphincter innervation. 
Electroencephalographic responses to bladder filling in humans have been demonstrated and have been utilized to investigate patients with brain disease and loss of bladder control (Kuru 1965; Raz and Bradley 1979).

The suggested method for future investigstion of patients with neurological dysfunction of the urinary bladder utilizing these methods would include:

1. The application of the sacral reflex evoked potentials (Bradley and Teague 1972; Dick et al. 1974) to detect interruption of the peripheral innervation of the urinary bladder and urethra.

2. The ultilization of cortical evoked potentials to determine if spinal cord pathways to the urinary bladder are intact.

3. Electroencepharography to define the nature of cerebro-cortical control of bladder function.

\section{References}

1) Andrew, J. \& Nathan, P.W. (1974) Lesions of the anterior frontal lobes and disturbances of micturition and defecation. Brain, 87, 233-262.

2) Bradley, W.E. (1977) Electroencephalography and bladder innervation. J. Urol., 118, 412-414.

3) Bradley, W.E. \& Teague, C.T. (1969) Cerebellar influence on the micturition reflex. Exp. Neurol., 23, 532-548.

4) Bradley, W.E. \& Teague, C.T. (1972) Electrophysiology of pelvic and pudendal nerves in the cat. Exp. Neurol., 35, 378-393.

5) Bradley, W.E. \& Scott, F.B. (1978) Physiology of the urinary bladder. Chapter IV, In: Campbell's Textbook of Urology, Vol. 1, W.B. Saunders Co., Philadelphia.

6) Dick, H., Bradley, W.E., Scott, F.B. \& Timm, G.W. (1974) Pudendal sexual reflexes. Urology, 3, 376-379.

7) Dill, R.C., Romiti, J.A. Kusske, J.A. \& Porter, R.W. (1977) Changes in discharge rate of epileptogenic foci during active and passive changes in urinary bladder pressure in cats. Exp. Neurol., 57, 532-548.

8) Gjone, R.S. (1963) Excitatory and inhibitory responses to stimulation of the cerebral cortex in the cat. Acta physiol. scand., 59, 337-348.

9) Imabayashi, K. \& Ohnuma, T. (1977) Brain Bladder - A new approach by cystometry. In: Eighteenth International Congress of Neurovegetative Research Praceedings, November 4-6, Tokyo. (Abstract)

10) Kuru, M. (1965) Nervous control of micturition. Physiol. Rev., 45, 426-465.

11) Langworthy, O.R. \& Kolb, L.C. (1933) The encephalic control of tone in the musculature of the urinary bladder. Brain, 56, 371-382.

12) Raz, S. \& Bradley, W.E. (1979) Neuromuscular dysfunction of the lower urinary tract. Chapter 35, In: Campbell's Textbook of Urology, Vol. II, W.B. Saunders Co., Philadelphia. 